\title{
Atazanavir urolithiasis without recent intake of atazanavir
}

\author{
Lithiase urinaire d'atazanavir sans prise récente \\ de ce médicament
}

\author{
Valery Brunel ${ }^{1}$ \\ Nathalie Massy ${ }^{2}$ \\ Benoit Malval $^{3}$ \\ ${ }^{1}$ Department of general biochemistry, \\ Charles-Nicolle University Hospital, \\ University of Rouen, France \\ ${ }^{2}$ Department of pharmacovigilance, \\ Charles-Nicolle University Hospital, \\ University of Rouen, France \\ ${ }^{3}$ Department of urology, \\ Charles-Nicolle University Hospital, \\ University of Rouen, France
}

Article received May 19, 2019,

accepted June 22, 2019

A urinary stone was observed on the follow-up CT urogram of a patient with HIV. The stone was removed by retrograde ureteroscopy. Then, laboratory analysis was performed by infrared spectroscopy. Eleven months previously, this patient had an episode of urolithiasis diagnosed on hematuria following a 12-year treatment with ritonavir-boosted atazanavir without study of crystalluria during those years. At this time, in front of radiolucency and known lithogenic effect of atazanavir, this one was replaced by darunavir. For this new episode, a brown friable urinary stone of $2 \mathrm{~mm}$ to $3 \mathrm{~mm}$ was observed. Radial crystallization was found on lithiasis section (figure 1A). The infrared spectrum of atazanavir was typical with a main narrow peak at $1724 \mathrm{~cm}^{-1}$ associated with typical peak at $3428 \mathrm{~cm}^{-1}$, without presence of other constituents (figure 1B). Laboratory analysis concluded atazanavir-related urolithiasis. Drug-related lithiasis is an uncommon etiology representing less than $1 \%$ of all causes. However, atazanavir represents more than a half of all cases of drug lithiasis. Atazanavir is excreted at a rate of $7 \%$ in unchanged form in urine. The mechanism of formation of atazanavir calculi involves urinary precipitation of pure atazanavir because of its weak solubility [1]. Two factors promoting the onset of urolithiasis were found in our patient. Hepatitis C co-infection, decreasing hepatic degradation of atazanavir, and low hydration (1L/day), increasing the final urinary concentration of atazanavir with an associated risk of precipitation. A similar case of atazanavir-related lithiasis far from discontinuation, has already been described. The feature of our case is the long delay, 12 years, before the first episode which usually occurs between 5 weeks and 6 years [2]. This case shows the importance of performing infrared spectroscopy to determine the underlying cause to guide clinical or therapeutic decision. Without analysis, this stone could have been mistakenly attributed to darunavir, known to form urinary crystals but whose capacity to form calculi has not yet been clearly demonstrated [1]. Cases of drug-related lithiasis should be systematically declared in national pharmacovigilance databases. 


\section{Biological pictures}

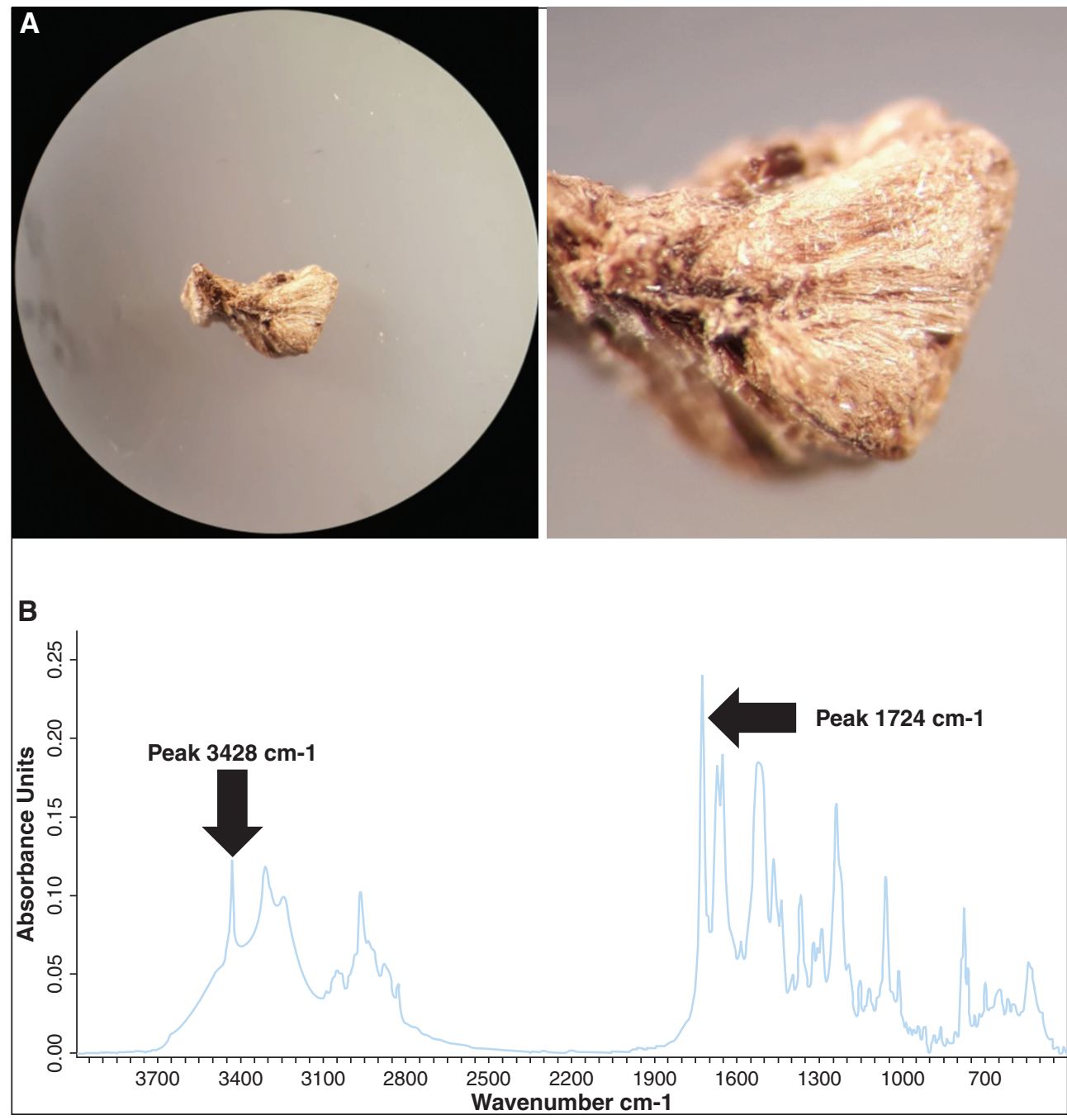

Figure 1. (A) Binocular loupe image of a fragment of stone. (B) Infrared spectrum of urolithiasis.

Acknowledgements. The authors are grateful to Nikki Sabourin-Gibbs, Rouen University Hospital, for reviewing the manuscript in english.

Conflict of interest: none of the authors has any conflict of interest to disclose.

\section{References}

1. Daudon M, Frochot V, Bazin D, Jungers P. Drug-induced kidney stones and crystalline nephropathy: pathophysiology, prevention and treatment. Drugs 2018; 78: 163-201.

2. Wang LC, Osterberg EC, David SG, Rosoff JS. Recurrent nephrolithiasis associated with atazanavir use. BMJ Case Rep 2014, Jan 8. 